\title{
Empirical Analysis of Traffic Bottleneck at Beijing Expressways
}

\author{
Sheng Jin, Dianhai Wang, and Dongfang Ma \\ College of Civil Engineering and Architecture, Zhejiang University, Hangzhou 310058, China \\ Correspondence should be addressed to Dongfang Ma; mdf2004@zju.edu.cn
}

Received 23 July 2013; Revised 16 September 2013; Accepted 16 September 2013

Academic Editor: Wuhong Wang

Copyright (C) 2013 Sheng Jin et al. This is an open access article distributed under the Creative Commons Attribution License, which permits unrestricted use, distribution, and reproduction in any medium, provided the original work is properly cited.

\begin{abstract}
The expressways in Beijing are confronted with more serious traffic congestions. Based on the survey data obtained from the typical sections at the expressways, the time dependent characteristics of traffic flow parameters were analyzed in detail and the data gap was found in this paper. The Fast Fourier Transform (FFT) method is proposed to transfer the data of traffic flow parameters for describing the fluctuation characteristics of traffic flow. Two methods of identification, the graph method and the control line method, were proposed as to the change time of traffic bottleneck forming and dissipating. The findings in this paper have already been applied in traffic management and ramp control at the expressways in Beijing.
\end{abstract}

\section{Introduction}

Nowadays, traffic congestions at the expressways have become more serious in Beijing, and the situation is deteriorating. One of the key reasons for traffic congestions is the merging conflicts of the entering and exiting traffic streams caused by the close distances from the upstream entrance ramp (or exit ramp) to the downstream one and the numerous entrance and exit ramps [1-3]. Traffic bottlenecks form easily in the conflict zones along the expressways, and the queue spilled over may result in congestion or even jam when traffic demand increases. Therefore, it is necessary to research on the traffic bottleneck for traffic management or ramp metering.

A prosperous literature can be observed on this topic. Bertini and Cassidy presented some traffic features at freeway bottlenecks. Observations from two-freeway bottlenecks in and near Toronto, Canada, indicated that average vehicles discharge rate from a queue could be $10 \%$ lower than the flow measured prior to the queue's formation. The present findings came by virtually comparing transformed curves of cumulative vehicle arrival number versus time and cumulative occupancy versus time measured at neighboring loop detectors [4]. Muoz and Daganzo researched the bottleneck mechanism of a freeway divaricating and found that an offramp queue may hamper freeway flow much more than an on-ramp bottleneck does [5]. By analyzing bottleneck formation on freeways, Das and Levinson identified "active bottleneck" locations on freeways and sections where bottlenecks occurred because of disturbances caused by downstream bottlenecks propagating backwards in the form of shockwaves [6]. Ogut and Banks worked on the stability of traffic flow at freeway bottlenecks [7]. Hu and Schonfeld developed a traffic simulation and optimization model to analyze traffic flow in large networks with severe queuing and to transfer traffic volume at bottlenecks [8].

Most of the work about traffic bottleneck mainly focused on the freeways, but a few on urban expressways, especially on those metropolises as in Beijing. Beijing Municipal Institute of City Planning and Design simply compared the traffic flow characteristics of freeways with those in some developed countries. It should be noted that many transport policies are under discussion in Beijing for congestion mitigation, for example, implementing the traffic congestion pricing [9, 10] and prompting the public transport systems [11]. In this paper, in order to analyze the traffic bottleneck formation, the time dependent characteristics of traffic flow parameters are analyzed in detail and the data gap is found on the basis of the survey data of typical sections chosen at Beijing expressways. The graph method and the control line method are put forward in order to assess the critical time of traffic state transition during bottleneck formation and dissipation. The former determines the critical time by adjusting curves of cumulative arrival vehicle number versus time and cumulative occupancy versus time. The latter designs an index, 


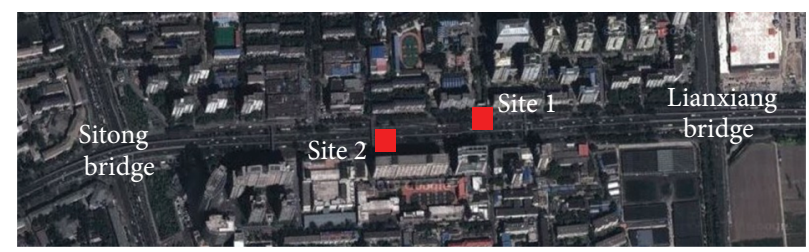

FIGURE 1: Map of the survey sites.

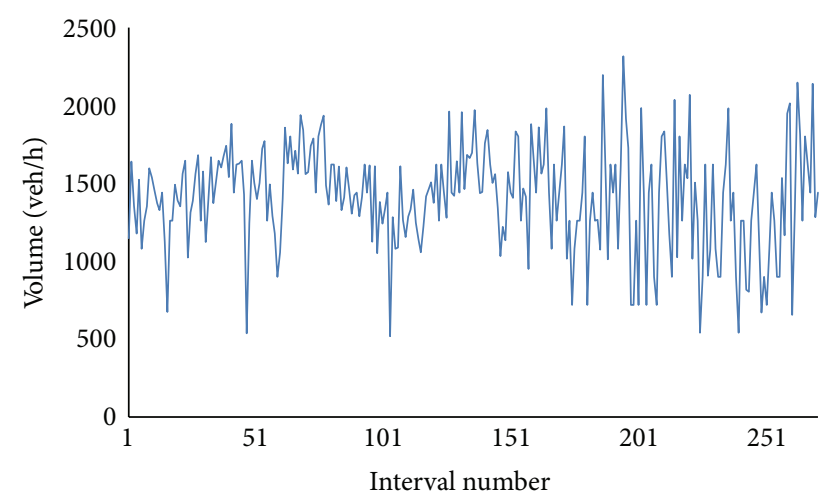

Figure 2: Volume on median lane at Site 1.

relative time occupancy which can better describe the traffic state, to calibrate the top and bottom boundary of the data gap according to the quality management principle.

\section{Traffic Flow Characteristics Analysis}

2.1. Time Dependent Characteristics. The field data (traffic volume, speed, and time occupancy) of a merge area and its upstream and downstream were obtained through the video survey between Sitong Bridge and Lianxiang Bridge in North Ring III at Beijing expressways from 7:00 a.m. to 10:00 a.m. (including morning peak hour) and from 16:00 p.m. to 19:00 p.m. (including evening peak hour) between June and July, 2010. To simplify, the site from Lianxiang Bridge to Sitong Bridge is noted as Site 1 , and the opposite direction as Site 2 (See Figure 1).

In data processing, 20 seconds was defined as a statistic time interval, and data were obtained including speed, traffic volume, and time occupancy. The time intervals $20 \mathrm{~s}, 30 \mathrm{~s}$, $1 \mathrm{~min}$, and $5 \mathrm{~min}$ are often used in traffic flow analysis. In order to describe the microcosmic fluctuation characteristics of traffic flow, we use the least time interval which can be provided by advanced transportation information system (ATIS). Here, speed is the harmonic average value of spot speed. Traffic volume is the vehicle number per hour per lane (veh/h/lane). Time occupancy is the ratio of time occupied by vehicles and statistical interval. The data are to analyze traffic flow characteristics and describe the process of traffic bottleneck forming and dissipating.

Figures 2-7 show the change of traffic flow parameters on the median lane at the survey section of Site 1 and Site 2 during the bottleneck dissipating. Figures 2 and 5 indicate traffic

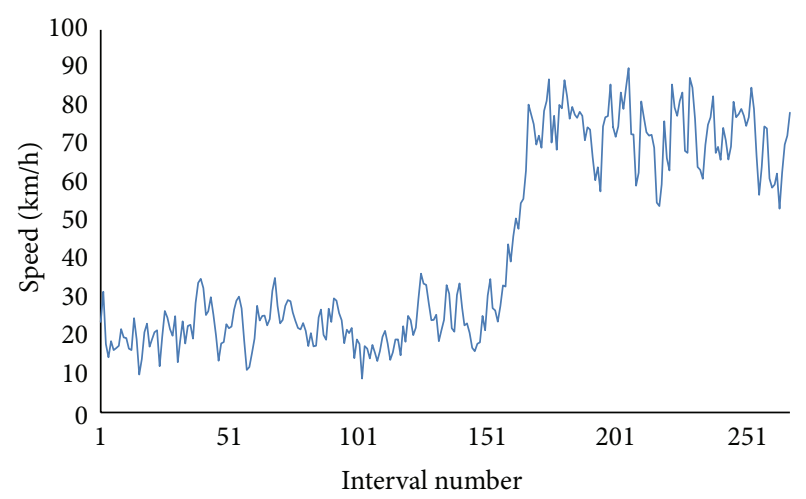

FIGURE 3: Speed on median lane at Site 1.

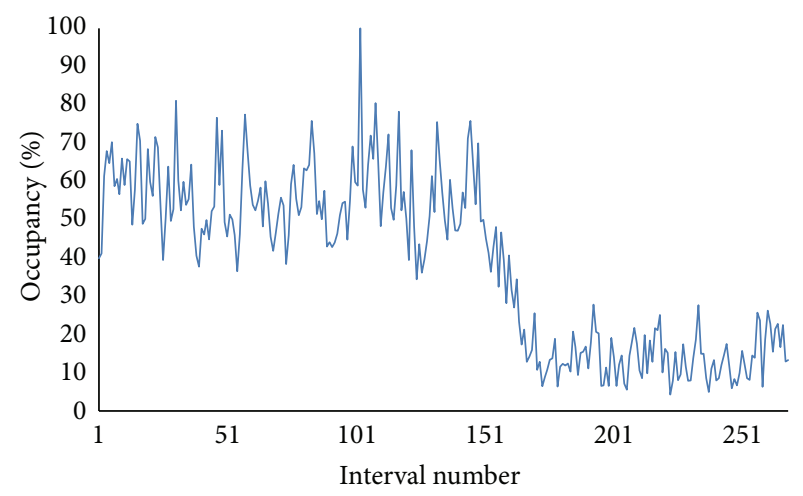

Figure 4: Occupancy on median lane at Site 1.

volume without obvious change from bottleneck forming to bottleneck dissipating. Figures 3 and 4 show that the speed (near $20 \mathrm{~km} / \mathrm{h}$ ) is lower and the time occupancy (near $60 \%)$ is higher when the traffic flow keeps in congestion state. The speed (near $75 \mathrm{~km} / \mathrm{h}$ ) goes up quickly, and on the contrary, the time occupancy (below 60\%) goes down quickly when the bottleneck dissipates. In Figures 6 and 7, the speed (near $75 \mathrm{~km} / \mathrm{h}$ ) is higher and the time occupancy (below 20\%) is lower when the traffic flow keeps in the normal state. The speed (near $20 \mathrm{~km} / \mathrm{h}$ ) goes down quickly and the time occupancy (upper 60\%) goes up quickly when traffic bottleneck forms, that is, the traffic flow changes from normal to congestion. When it is changed from congestion to normal, the speed goes up and the time occupancy goes down quickly, respectively. Data from the two sites indicates that the curves of speed and time occupancy can reflect the state transition from congestion to noncongestion (or the reverse), but the 


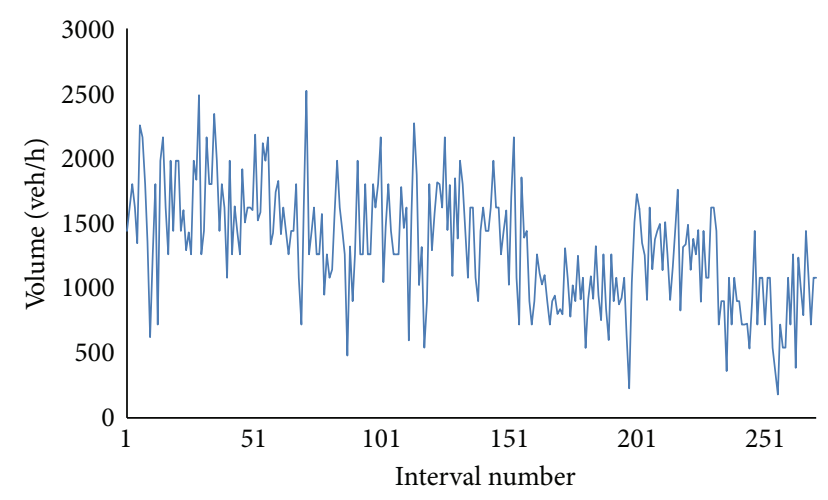

FIGURE 5: Volume on median lane at Site 2.

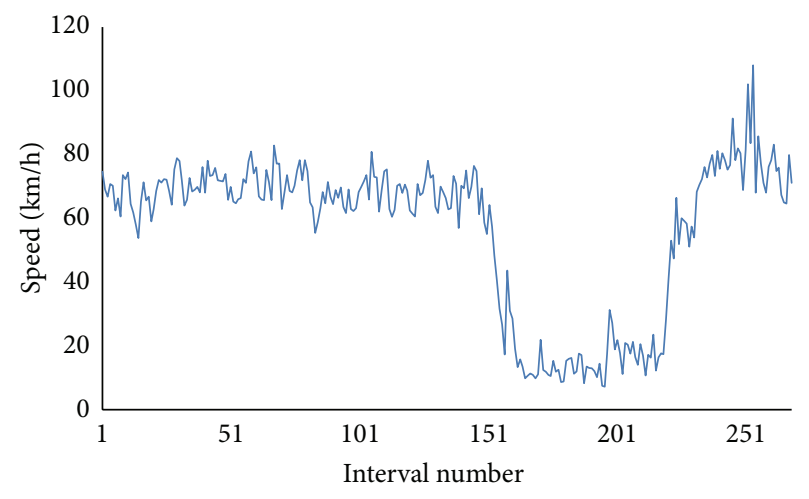

FIgURE 6: Speed on median lane at Site 2.

curve of traffic volume does not. The change rule of traffic flow parameters is similar for the middle lane and shoulder lane.

Figures 8,9 , and 10 are, respectively, the volume versus speed scatter plot, the occupancy versus volume scatter plot, and the occupancy versus speed scatter plot on all the lanes of the merge area downstream at Site 1.

In Figures 8-10, it is found that data pots cluster into two regions, one represents congestion data, while the other represents noncongestion data, and there is a space with sparse data, without any data in between. The space is defined as data gap [12-18]. The data gap also reflects the transition from one traffic state to another.

2.2. Fluctuation Characteristics. From the time dependent characteristics figures (see Figures 2-7) of traffic flow parameters, it is found that these parameters fluctuate with time when the traffic state is in congestion (see Figures 11, 12, and 13). Some fluctuation characteristics of speeds at freeways had been studied [19-21]. The data obtained at Beijing expressways by us reveal the same characteristics. The fluctuation is regarded as the waves consisting of a series of overlapped simple harmonic oscillation. We can use some periodic functions like sine, cosine, or both of them to fit the field data. In order to simplify the calculation, the sine functions are adopted to fit the fluctuation characteristics of the traffic flow parameters (see (1)). The Fast Fourier

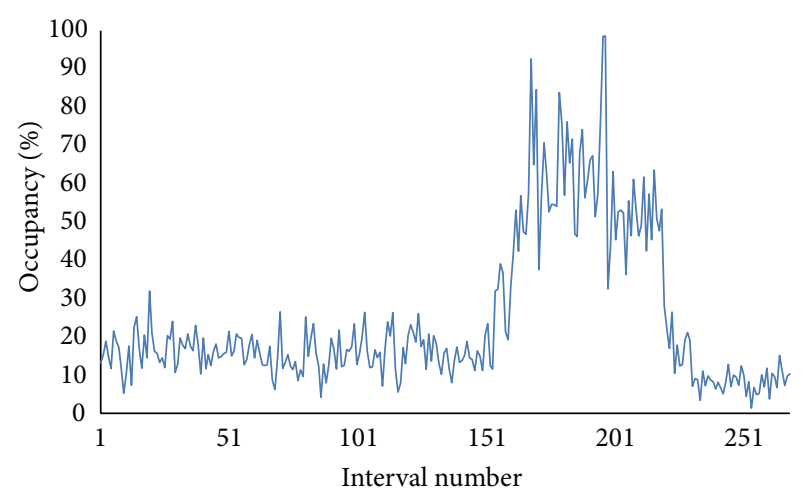

Figure 7: Occupancy on median lane at Site 2.

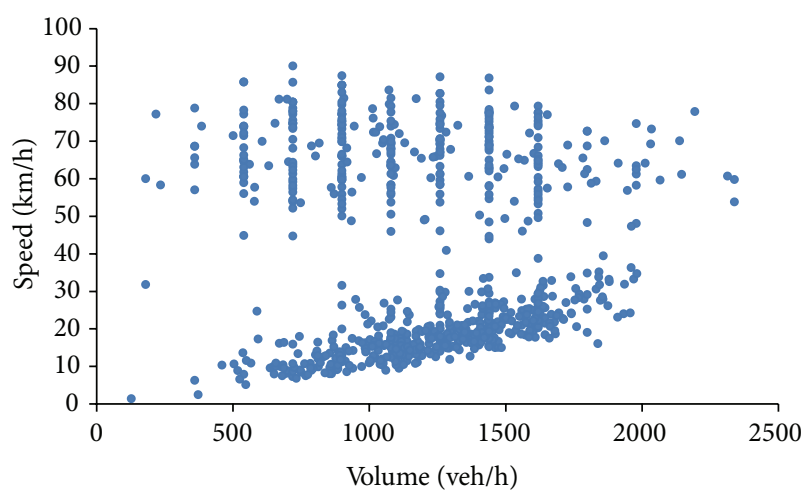

FIGURE 8: Volume-speed plot at Site 1.

Transform (FFT) method is used to transfer the data of traffic flow parameters (data source from Figures 2-4) from time domain to frequency domain. Then, the simple harmonic oscillation with the largest amplitudes is folded to fit the field data at the object of the minimized relative error. Consider

$$
f(t)=a+\sum_{i=1}^{n} A_{i} \sin \left(\omega_{i} t+\varphi_{i}\right)
$$

where $a$ is the fluctuation mean value of the traffic flow parameters, $A_{i}$ is the amplitude, $\omega_{i}$ is the angular velocity, $\varphi_{i}$ is the prime phase angle, $t$ is the interval number, and $n$ is the number of sine functions.

Table 1 shows the fitting function values (the amplitudes, angular velocities, and phase angles) of traffic flow parameters obtained from the middle lane of the merge area upstream at Site 2 in congestion state.

From Table 1, with the same angular velocity, satisfactory functions are obtained to describe the fluctuations of traffic volume, speed, and time occupancy. The research results show that there are similar fluctuation rules on the median lane and shoulder lane. Except for the merge area, the same fluctuation facts are found at the other locations. Therefore, the conclusion drawn is that the congested traffic flow parameters (traffic volume, speed, and time occupancy) fluctuate periodically with time, and the periodical fluctuations of these parameters are intrinsic property of urban expressway system. 


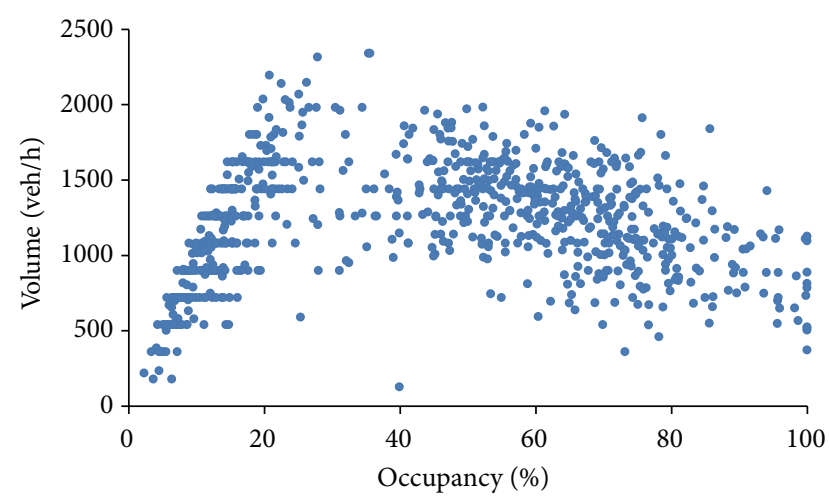

Figure 9: Occupancy-volume plot at Site 1.

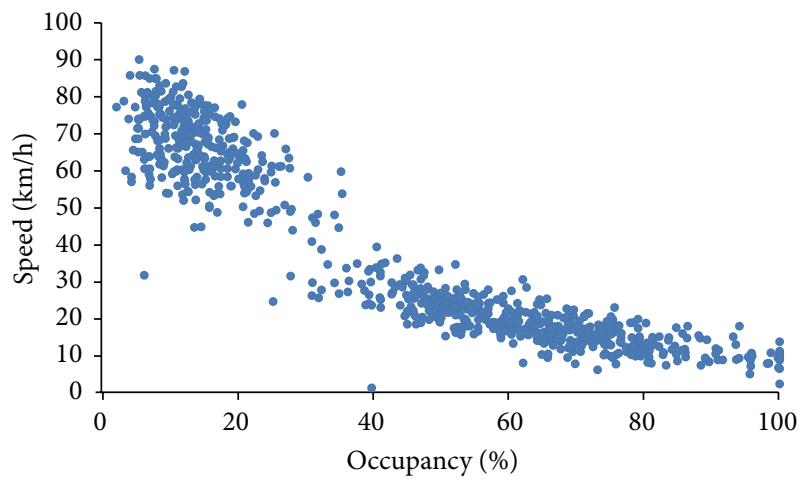

FIGURE 10: Occupancy-speed plot at Site 1.

\section{Critical Time Identification}

The critical time dividing traffic states is of importance to describe the bottleneck characteristic and is of great value for the traffic control system of urban expressways. Two methods are proposed to define it: the graph method and the control line method.

3.1. Graph Method. According to the traffic flow theory, traffic volume changes with time in accordance with time occupancy when traffic flow is in noncongestion $[19,22]$. The accumulative vehicle number curve and the accumulative time occupancy curve can be properly adjusted to make them overlap in the same coordinate plane. But, the time occupancy ascends quickly and the traffic volume descends when traffic flow changes from noncongestion to congestion. Therefore, the slope of accumulative time occupancy curve increases, and that of accumulative vehicle number curve decreases. So, there must be a point where the two adjusted curves start to furcate and the trajectories go in different directions. Then, the time corresponding to the furcated point is the critical time in traffic state transition. During traffic flow resuming normal state, the two adjusted curves gradually come close before they merge. The time corresponding to the joining point is the critical time when traffic state changes from congestion to normal.

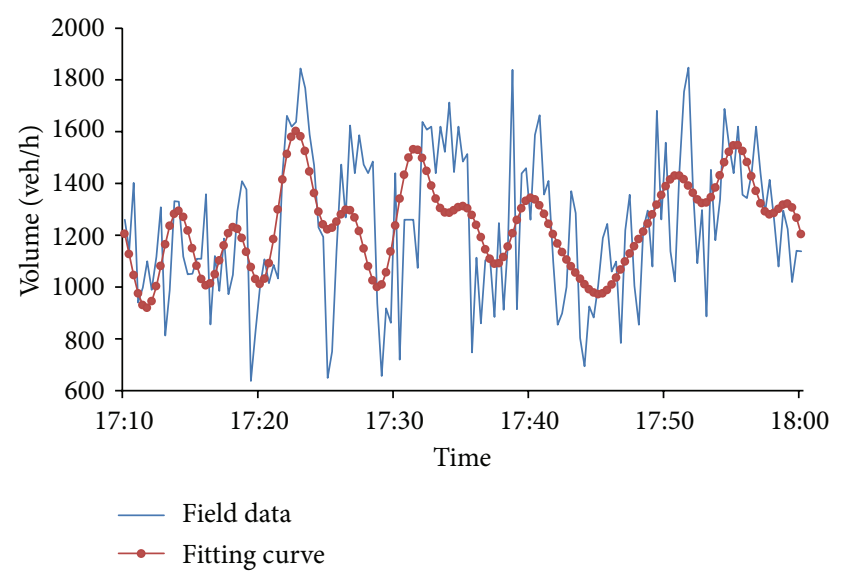

FIGURE 11: Volume fluctuation characteristic on middle lane at Site 2 .

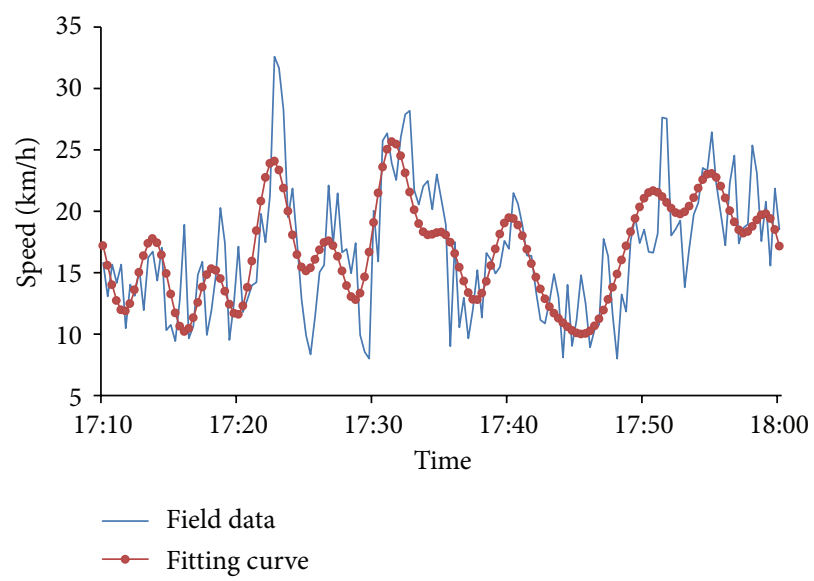

FIGURE 12: Speed fluctuation characteristic on middle lane at Site 2.

TABLE 1: The coefficient value of fitting functions on traffic flow parameters in congestion.

\begin{tabular}{|c|c|c|c|c|c|c|}
\hline & 1 & 2 & 3 & 4 & 5 & 6 \\
\hline \multicolumn{7}{|c|}{ Volume } \\
\hline$A_{i}$ & 0.66 & 0.57 & 0.35 & 0.60 & 0.47 & 0.42 \\
\hline$\omega_{i}$ & 0.08 & 0.13 & 0.21 & 0.25 & 0.46 & 0.50 \\
\hline$\varphi_{i}$ & 2.96 & 3.67 & -0.23 & 3.56 & 2.56 & 1.21 \\
\hline$a$ & \multicolumn{2}{|c|}{6.88} & \multicolumn{2}{|c|}{ MRE } & \multicolumn{2}{|c|}{$10.38 \%$} \\
\hline \multicolumn{7}{|l|}{ Speed } \\
\hline$A_{i}$ & 0.95 & 0.53 & 0.59 & 0.61 & 0.57 & 0.39 \\
\hline$\omega_{i}$ & 0.08 & 0.13 & 0.21 & 0.25 & 0.46 & 0.50 \\
\hline$\varphi_{i}$ & 2.77 & 3.75 & 0.21 & 3.61 & 2.74 & 1.21 \\
\hline$a$ & \multicolumn{2}{|c|}{4.70} & \multicolumn{2}{|c|}{ MRE } & \multicolumn{2}{|c|}{$12.09 \%$} \\
\hline \multicolumn{7}{|c|}{ Occupancy } \\
\hline$A_{i}$ & 0.08 & 0.03 & 0.03 & 0.03 & 0.04 & 0.03 \\
\hline$\omega_{i}$ & 0.08 & 0.13 & 0.21 & 0.25 & 0.46 & 0.50 \\
\hline$\varphi_{i}$ & -0.34 & 0.53 & 3.46 & 0.36 & -0.21 & 4.54 \\
\hline$a$ & \multicolumn{2}{|c|}{0.68} & \multicolumn{2}{|c|}{ MRE } & \multicolumn{2}{|c|}{$7.52 \%$} \\
\hline
\end{tabular}

The method to affirm the critical time of traffic state is to adjust the curves of cumulative vehicle arrival numbers 


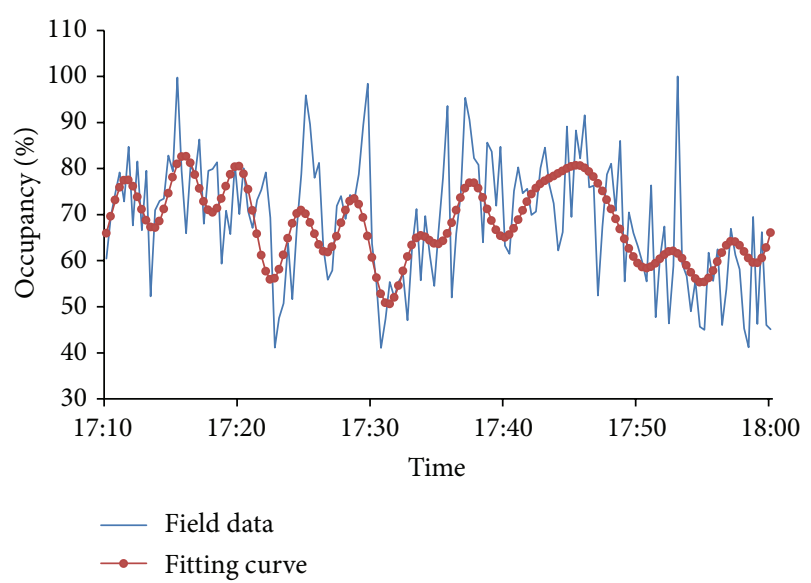

Figure 13: Occupancy fluctuation characteristic on middle lane at Site 2.

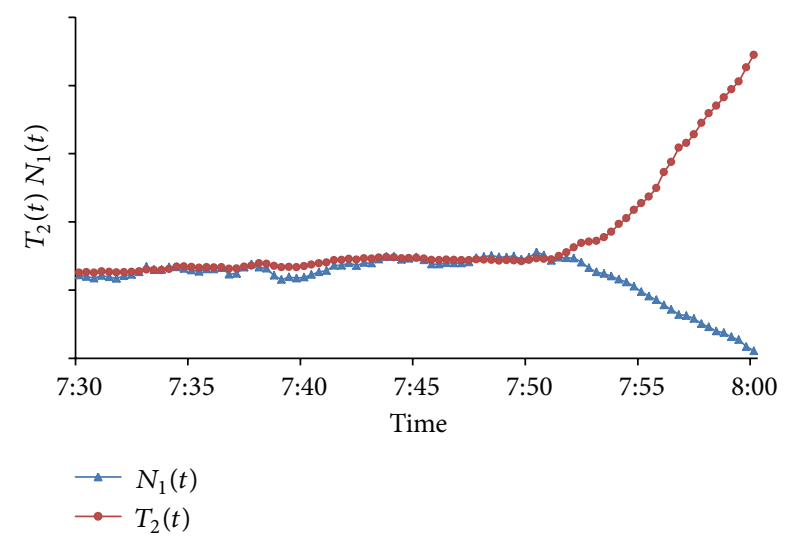

FIgURE 14: Critical time of traffic state change of median lane at Site 1.

and cumulative time occupancy. The adjusted accumulative vehicle number is

$$
N_{1}(t)=N_{0}(t)-q t^{\prime},
$$

where $N_{1}(t)$ is the adjusted accumulative vehicle number at time $t, N_{0}(t)$ is the accumulative vehicle number at time $t, q$ is a flow to be calibrated, and $t^{\prime}$ is the time starting from a point on the time axis when traffic is in noncongestion. Equation

(2) is the change of $N_{1}(t)$ decided by the flow.

The adjusted accumulative time occupancy is

$$
T_{1}(t)=T_{0}(t)-b t^{\prime}
$$

where $T_{1}(t)$ is the adjusted accumulative time occupancy at time $t, T_{0}(t)$ is the accumulative time occupancy at time $t$, and $b$ is a coefficient. In (3), the change of $T_{1}(t)$ is decided by the coefficient $b$.

In order to make the two adjusted curves overlap in the same coordinate plane when traffic is in noncongestion, the adjusted curve of accumulative time occupancy is amplified by $k$ times. Equation (3) is modified as

$$
T_{2}(t)=k\left[T_{0}(t)-b t^{\prime}\right] .
$$

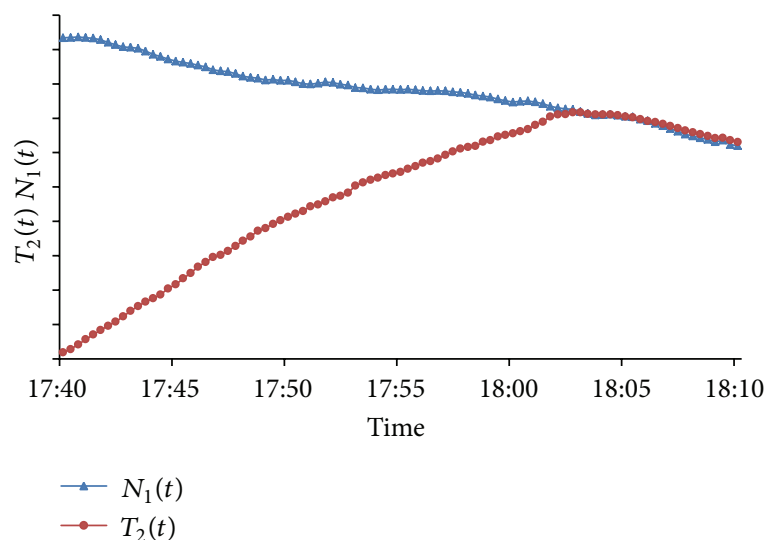

FIGURE 15: Critical time of traffic state on middle lane at Site 2.

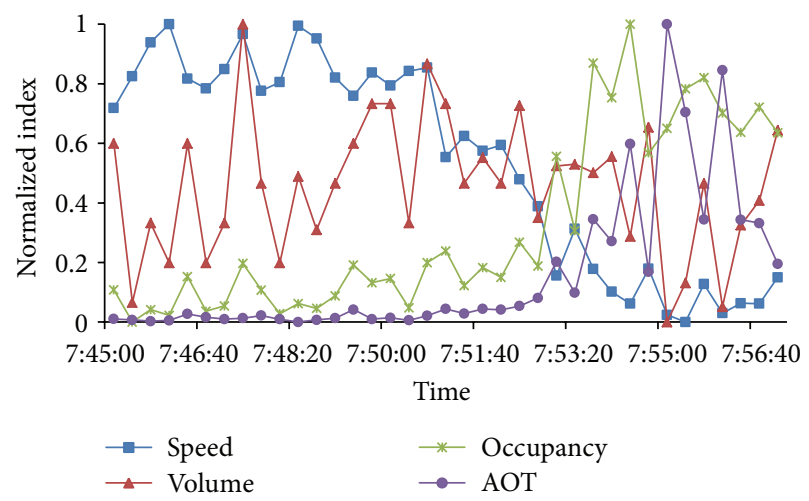

FIGURE 16: Normalization index on median lane at Site 1.

Based on the analysis above, (2) and (4) are applied to plot the two adjusted curves and decide the critical time corresponding to the furcate point or the joining point.

The procedures of affirming the critical time of traffic state are as follows:

(1) confirm the approximate time of traffic state transition according to the curve of occupancy versus time;

(2) select the original data about the approximate time;

(3) confirm the coefficients $q, b$, and $k$ on the basis of step 2 ;

(4) plot the adjusted curves of accumulative vehicle number and accumulative time occupancy according to (2) and (4);

(5) affirm the critical time of traffic state in terms of the furcate and joining points.

Here, the basic principle to select $q, b$, and $k$ needs to be specified. When traffic flow changes from normal to congestion, the mean values of traffic volume and time occupancy in the normal situation are defined as $q, b$, and $k$ are between 10 and 20 in general. When traffic flow changes from congestion to normal, $q$ and $k$ are identical to the above results, but $b$ equals 0.3 [23-25].

The graph method is applied to affirm the critical time by the survey data $(7: 30 \sim 8: 00)$ on the median lane of 


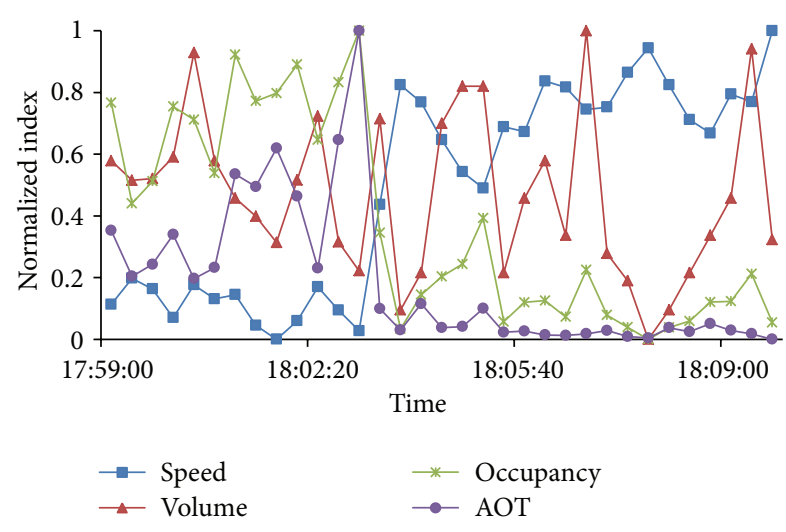

FIgURE 17: Normalization index on shoulder lane at Site 2.

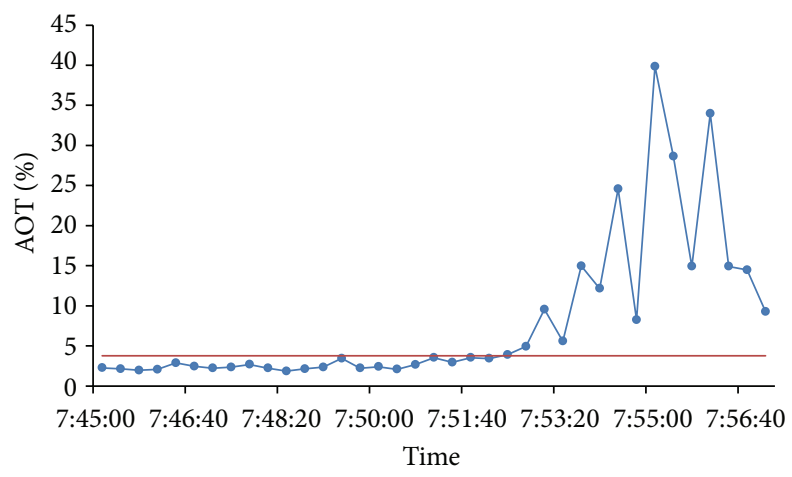

Figure 18: Management plots on median lane at Site 1.

the merge area downstream at Site 1. Here, $q$ equals 1455 (veh/h), $b$ equals 0.15 , and $k$ equals 15 . The adjusted curves of accumulative time occupancy and accumulative vehicle number are shown in Figure 14. The furcate time of two curves is about 7:51:20, which becomes the turning point, where traffic flow changes from normal to congestion, that is, traffic bottleneck is forming. The same work is done to affirm the critical time by the survey data $(17: 40 \sim 18: 20)$ on the middle lane of the merge area upstream at Site 2. Here, $q$ equals 1530 (veh/h), $b$ equals 0.3 , and $k$ equals 15 . The adjusted curves of accumulative time occupancy and accumulative vehicle number are shown in Figure 15. The joining time of the curves is at 18:03:00. From this point, traffic flow changes from congestion to normal, that is, traffic bottleneck has dissipated. The analysis result accords with video observation.

3.2. Control Line Method. According to the present research, it is inaccurate to use a single traffic flow parameter to determine the state transition at expressways. A compound index, average occupancy time (AOT) is designed to affirm the critical time of traffic state. The expression of index AOT is

$$
\mathrm{AOT}=\frac{\sum t_{i}}{q} .
$$

Here, $t_{i}$ is the time of number $i$ vehicle passing through the detectors, and $q$ is traffic volume.

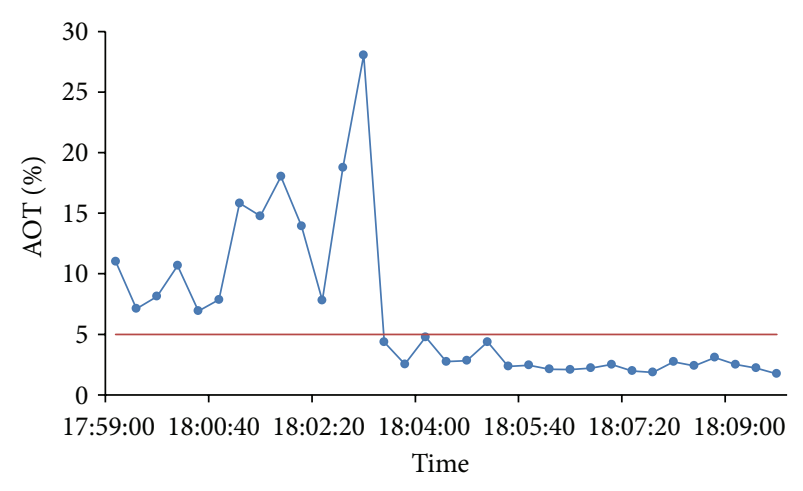

FIGURE 19: Management plots on shoulder lane at Site 2.

It is well known that the detector offers time occupancy parameter rather than the parameter $\sum t_{i}$ [26]. But time occupancy is equal to $\sum t_{i}$ divided by $T$; that is, $o=\sum t_{i} / T$. Therefore, the index AOT becomes AOT which equals to time occupancy divided by traffic volume; that is, $\mathrm{AOT}=o / q$.

Figures 16 and 17 show the curves of speed versus time, volume versus time, occupancy versus time, and AOT versus time after normalization processing at different site respectively. The index AOT is more stable than the single index (such as time occupancy, and speed) in normal situation, and it has an obvious change when traffic state changes from one to another. So the index AOT adapts to describe the transition of traffic state.

According to the quality management principle, traffic is regarded as a production process following normal distribution. When traffic state is normal, it can be regarded as a normal state, otherwise an abnormal state. By calculating $\mathrm{AOT}$, a quality management diagram can be drawn (AOT $-t)$ to determine the transition of traffic state. If the AOT data falls in the range of $(\mu \pm 3 \sigma)$, it indicates that traffic state is of noncongestion. If three consecutive data are more than the upper boundary $(\mu+3 \sigma)$, traffic state will change to congestion at the time. On the contrary, when traffic state is firstly in congestion, if three consecutive data points were lowered to the upper boundary $(\mu+3 \sigma)$, then this indicates that traffic state returns to noncongestion.

The detailed procedure to affirm the transition of traffic state is listed as follows.

(1) Select data in normal situation to confirm mean value $\mu$ and square error $\sigma$ of AOT, and the data sample number exceeds 45 if adopting $20 \mathrm{~s}$ as time interval because the observed period is not less than 15 minutes in general.

(2) Calculate the boundary value $(\mu \pm 3 \sigma)$ of AOT according to step 1 . Here, the lower boundary $(\mu-3 \sigma)$ of AOT is not to be considered because all traffic states below the boundary $(\mu+3 \sigma)$ are regarded as normal state.

(3) Determine traffic state by analyzing the index value of AOT continuously. If three consecutive data points exceed the upper boundary $(\mu+3 \sigma)$, traffic state will change from normal to congestion at the time. 
If three consecutive data points are lower to the upper boundary $(\mu+3 \sigma)$, traffic flow returns to noncongestion.

Taking, for example, the field data on the median lane of the merge area downstream at Site 1 and on the shoulder lane of the merge area upstream at Site 2, then the control line method is validated (see Figures 18 and 19).

In Figure 18, 50 points in normal situation are chosen to get $\mu$ and $\sigma$. If $\mu$ is 0.0276 and $\sigma$ is 0.0036 , then $\mu+3 \sigma$ is 0.0384 . The selected time period is $7: 47 \sim 7: 58$. The transition time from normal to congestion is 7:51 by applying the control line method. In Figure 19, 50 points in normal situation are selected to get $\mu$ and $\sigma$. If $\mu$ is 0.0256 and $\sigma$ is 0.0080 , then $\mu+$ $3 \sigma$ is equal to 0.0496 . The selected time period is 17:59 18:12. The time of traffic flow changing from congestion to normal is 18:03 by applying the control line method. The outcome accords with the video observation and the graph method.

\section{Conclusions}

Based on the survey data of the section chosen from Beijing expressways, the time dependent characteristics of the traffic flow parameters are analyzed and the data gap is found. Two methods are proposed: the graph method and the control line method in order to affirm the critical time of traffic state when traffic flow changes from congestion to normal situation (or vice versa). The research outcome shows that the two methods are feasible in accordance with the result of video observation. The findings of the paper have been applied in the control plan of Beijing expressways. Future research will focus on using the field data from different cities to calibration and validation of the proposed models.

\section{Conflict of Interests}

The authors declare that there is no conflict of interests regarding the publication of this paper.

\section{Acknowledgment}

This work was supported by the National Natural Science Foundation of China (nos. 51278454, 51208462, and 61304191).

\section{References}

[1] X. Qu, Y. Kuang, E. Oh, and S. Jin, "Safety evaluation for expressways: a comparative study for macroscopic and microscopic indicators," Traffic Injury Prevention, 2013.

[2] Q. Meng, X. Qu, K. T. Yong, and Y. H. Wong, "QRA modelbased risk impact analysis of traffic flow in urban road tunnels," Risk Analysis, vol. 31, no. 12, pp. 1872-1882, 2011.

[3] W. Wuhong, B. Klaus, and W. Geert, "Discrete dynamics in transportation system," Discrete Dynamics in Nature and Society, vol. 2013, Article ID 234970, 2 pages, 2013.

[4] R. L. Bertini and M. J. Cassidy, "Some observed queue discharge features at a freeway bottleneck downstream of a merge," Transportation Research A, vol. 36, no. 8, pp. 683-697, 2002.
[5] J. C. Muoz and C. F. Daganzo, "The bottleneck mechanism of a freeway diverge," Transportation Research A, vol. 36, no. 6, pp. 483-505, 2002.

[6] S. Das and D. Levinson, "Queuing and statistical analysis of freeway bottleneck formation," Journal of Transportation Engineering, vol. 130, no. 6, pp. 787-795, 2004.

[7] K. S. Ogut and J. H. Banks, "Stability of freeway bottleneck flow phenomena," Transportation Research Record, no. 1934, pp. 108115, 2005.

[8] Y.-C. Hu and P. Schonfeld, "Optimizing traffic diversion around bottlenecks," Transportation Research Record, pp. 22-27, 1984.

[9] Z. Liu, Q. Meng, and S. Wang, "Speed-based toll design for cordon-based congestion pricing scheme," Transportation Research C, vol. 31, pp. 83-98, 2013.

[10] Z. Liu and Q. Meng, "Modelling transit-based park-and-ride services on a multimodal network with congestion pricing schemes," International Journal of Systems Science, 2012.

[11] Z. Liu, Y. Yan, X. Qu, and Y. Zhang, "Bus stop-skipping scheme with random travel time," Transportation Research C, vol. 35, pp. 46-56, 2013.

[12] L. C. Edie, "Car-following and steady-state theory for noncongested traffic," Operations Research, vol. 9, pp. 66-76, 1961.

[13] J. S. Drake et al., "Statistical analysis of density hy-potheses," Highway Transportation Research, vol. 154, pp. 53-87, 1967.

[14] A. Ceder, "A deterministic traffic flow model for the two-regime approach," Transportation Research Record, no. 567, pp. 16-30, 1976.

[15] J. C. Munoz and C. F. Daganzo, "Structure of the transition zone behind freeway queues," PATH Working Paper UCB-ITSPWP-2000-24, Institute of Transportation Studies, University of California, Berkeley, Calif, USA, 2000.

[16] M. Koshi, M. Iwasaki, and I. Ohkura, "Some findings and an overview on vehicular flow characteristics," Proceedings of the 8th International Symposium on Transportation and Traffic Theory, pp. 403-426, 1983.

[17] Q. Meng and X. Qu, "Estimation of rear-end vehicle crash frequencies in urban road tunnels," Accident Analysis and Prevention, vol. 48, pp. 254-263, 2012.

[18] S. Jin, X. Qu, and D. Wang, "Assessment of expressway traffic safety using gaussian mixture model based on time to collision," International Journal of Computational Intelligence Systems, vol. 4, pp. 1122-1130, 2011.

[19] H. Nathan, Monograph on Traffic Flow Theory, The Federal Highway Administration (FHWA), 1996.

[20] B. S. Kerner, The Physics of Traffic, Springer, 2004.

[21] W. Wang, W. Zhang, H. Guo, H. Bubb, and K. Ikeuchi, "A safetybased approaching behavioural model with various driving characteristics," Transportation Research C, vol. 19, no. 6, pp. 1202-1214, 2011.

[22] Q. Meng and X. Qu, "Uncertainty propagation in quantitative risk assessment modeling for fire in road tunnels," IEEE Transactions on Systems, Man and Cybernetics C, vol. 42, pp. 14541464, 2012.

[23] Transportation Research Board, Highway Capacity Manual, National Research Council, 2002.

[24] M. J. Cassidy and R. L. Bertini, "Some traffic features at freeway bottlenecks," Transportation Research B, vol. 33B, no. 1, pp. 2542, 1999.

[25] R. M. Shanteau, "Using cumulative curves to measure saturation flow and lost time," Institute of Transportation Engineers Journal, vol. 58, no. 10, pp. 27-31, 1988.

[26] A. D. May, Traffic Flow Fundamentals, Library of Congress Cataloging in Publication Data, 1990. 


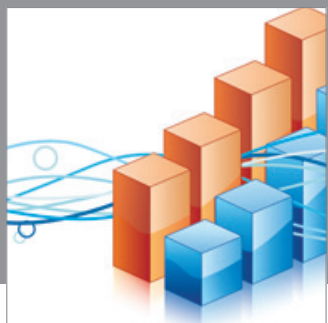

Advances in

Operations Research

mansans

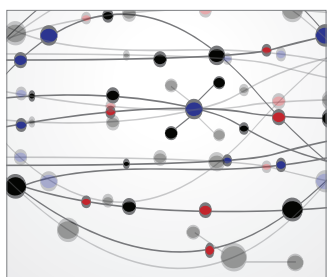

The Scientific World Journal
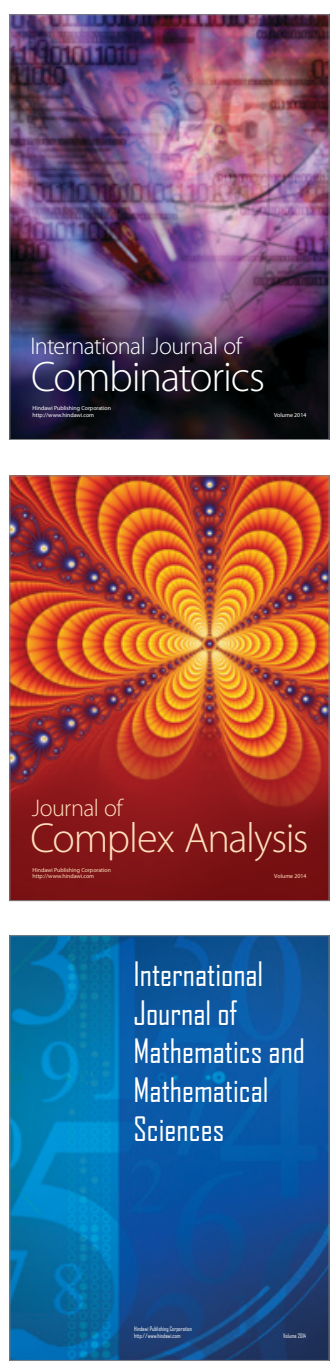
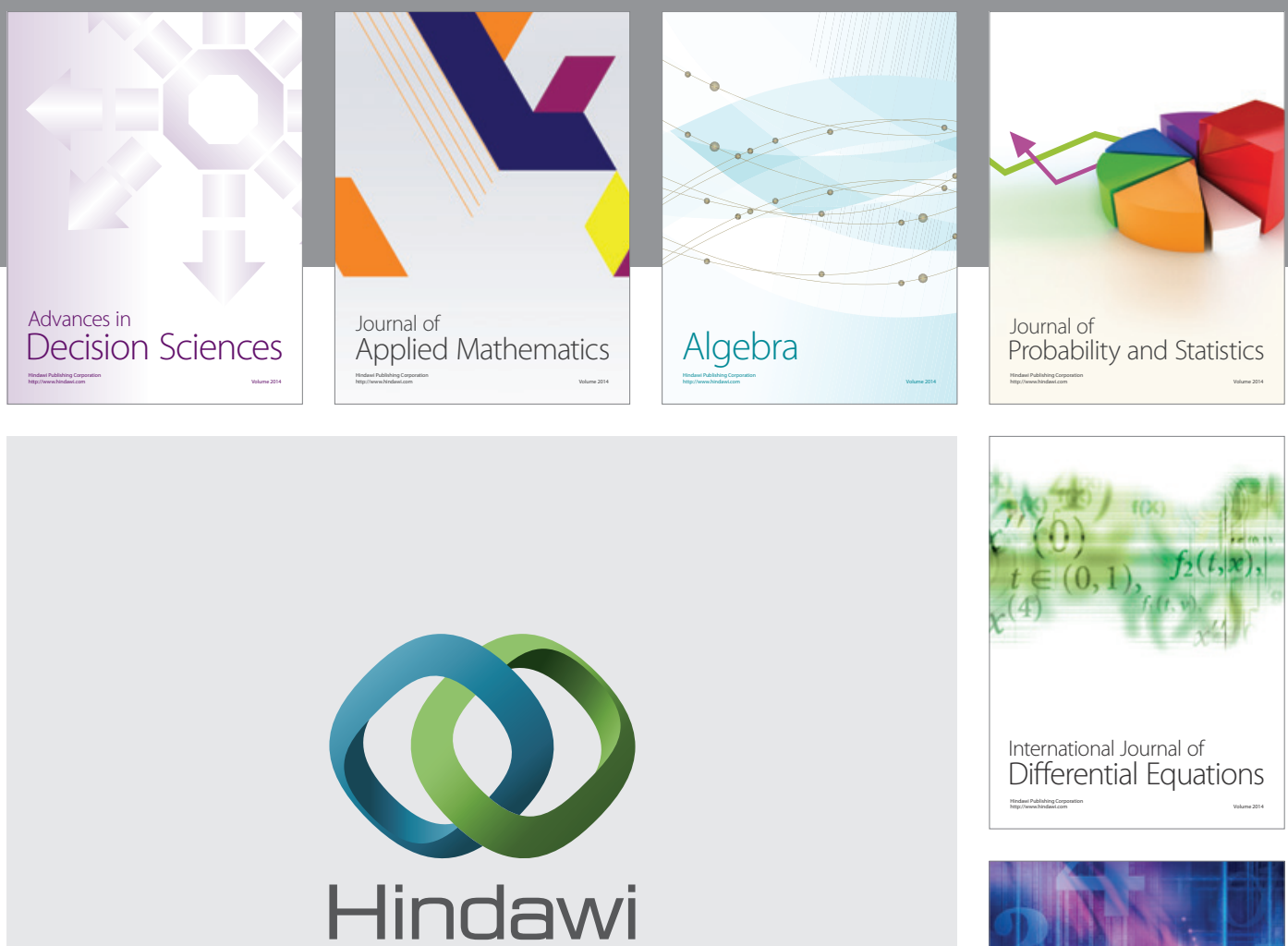

Submit your manuscripts at http://www.hindawi.com
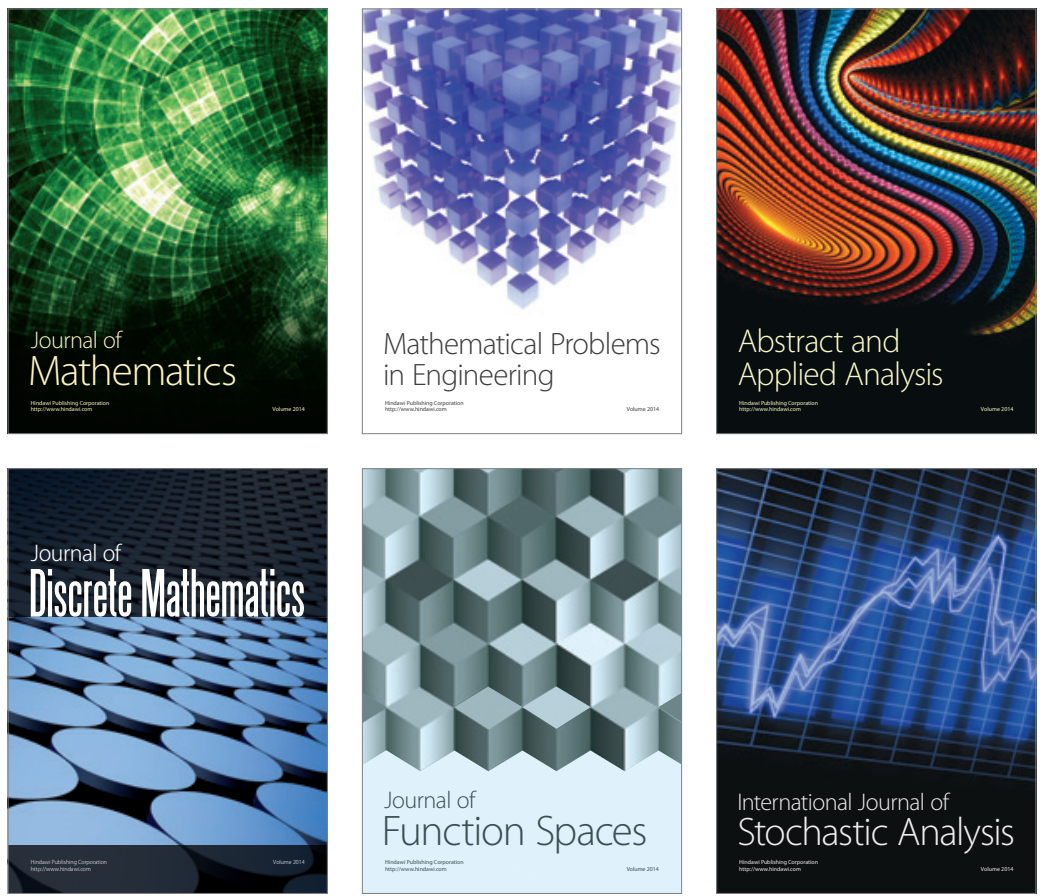

Journal of

Function Spaces

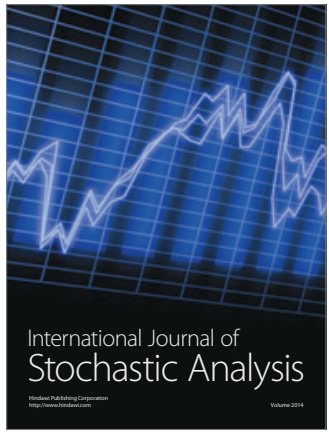

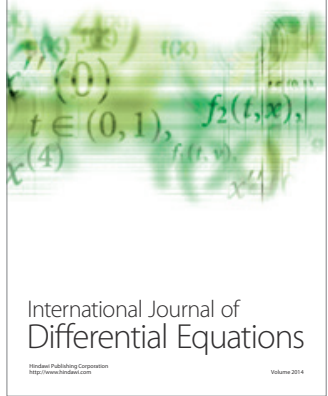
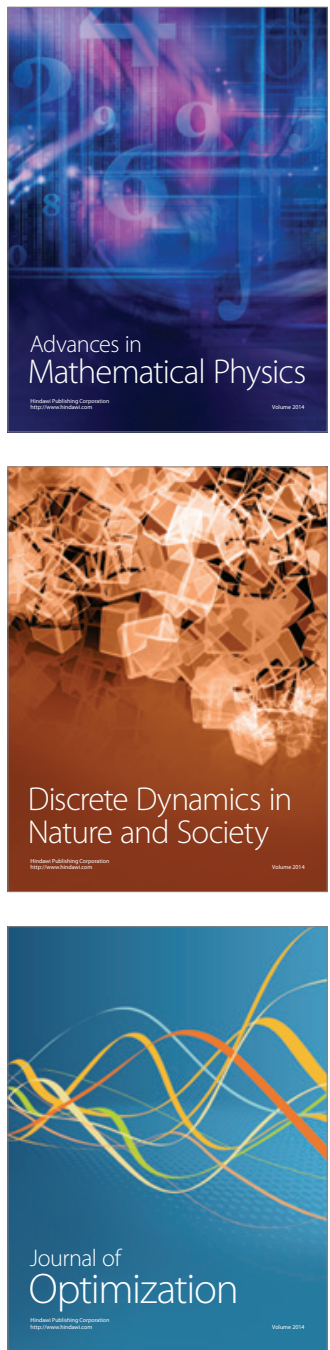